\title{
Taxionomia de Vídeos para o Ensino de Matemática
}

\author{
Rosiane de Jesus Santos ${ }^{1}$, Eduardo Barrére ${ }^{2}$ \\ ${ }^{1}$ Programa de Pós-Graduação em Educação Matemática - Universidade Federal de Juiz \\ de Fora (UFJF). \\ ${ }^{2}$ Departamento de Ciência da Computação - Universidade Federal de Juiz de Fora
} (UFJF) rosijs7@yahoo.com.br, eduardo.barrere@ice.ufjf.br

\begin{abstract}
This paper aims to present a proposal for a Taxonomy of math videos to support teachers in decision making in the evaluation and selection of instructional videos in the context of mathematics education. For this purpose a study of the objectives of Mathematics for Elementary Education II described by the NCP was done by Bloom's Taxonomy in order to identify and develop goals and skills related to the cognitive domain characteristics. There was also an experiment with math videos to identify key features for the taxonomy. Research has show the proposed taxonomy of videos showing the basic aspects that compose it.
\end{abstract}

\begin{abstract}
Resumo: Este artigo visa apresentar uma proposta de Taxionomia de vídeos de matemática para apoiar os professores na tomada de decisão na avaliação e seleção de vídeos didáticos no contexto da educação matemática. Para este propósito foi realizado um estudo sobre os objetivos da Matemática para o Ensino Fundamental II descrito pelo PCN através da Taxionomia de Bloom, a fim de identificar e explorar características relacionadas aos objetivos e habilidades do domínio cognitivo. Também foi feito um experimento com vídeos de matemática para identificar características fundamentais para a taxionomia. A pesquisa vem mostrar a proposta de taxionomia de vídeos evidenciando os aspectos elementares que a compõe.
\end{abstract}

\section{Introdução}

A inserção das tecnologias no ensino da matemática vem contribuindo cada vez mais com o processo ensino e aprendizagem realizado pelos professores e alunos. Dentre essas tecnologias está à mídia vídeo, que com o grande acesso a internet, vem se destacando como material didático no ensino, apresentando um conteúdo constituído por imagens, sons, texto escrito, efeitos, citações, dentre outros elementos que o compõe, envolvendo diferentes modos de linguagens [Moram 2005].

No ensino da matemática o vídeo pode favorecer na compreensão dos conteúdos através de diferentes abordagens, bem como a possibilidade de desenvolver a criatividade, o interesse e a imaginação dos alunos [Silva 2011]. Entretanto, o uso do vídeo no ensino, muitas das vezes, acontece sem a preocupação de analisar o tipo e a 
qualidade do material que esta sendo empregado nas atividades. Alguns professores ao não exercer esta prática de analise, acabam desconhecendo as funções do vídeo que podem desenvolver o aprendizado do aluno.

$\mathrm{Na}$ literatura existem alguns trabalhos como Ferrés (1996), Gomes (2008) que buscam sistematizar características dos vídeos no que tange ao seu uso didático em sala de aula, contudo, não foram encontrados trabalhos referenciando a avaliação e seleção deste tipo de material, especificamente para o ensino de matemática.

Desta forma o artigo vem apresentar uma proposta de classificação de vídeos didáticos de matemática para apoiar a seleção e avaliação deste material realizada pelos professores. A avaliação do material audiovisual constitui em criar condições ou referências para que o professor se baseie no momento de seleção e verificação da qualidade do vídeo.

A taxionomia de vídeos foi construída através de realização de experimentos de análise de vídeos, de um estudo da taxionomia de Bloom sobre objetivos educacionais embasado no domínio cognitivo e nos objetivos gerais para o ensino de matemática do Ensino Fundamental II descrito pelo Parâmetro Curricular Nacional (PCN).

Com esta taxionomia espera-se no futuro realizar uma pesquisa de campo para verificar sua eficácia que contribuirá para a elaboração do guia de seleção e avaliação de vídeos didáticos destinados aos professores de matemática.

\section{Os Objetivos da Matemática a partir da Taxionomia de Bloom}

A taxionomia de Bloom é considerada como "um sistema de classificação de objetivos educacionais" [Prata and Nascimento 2007]. Os objetivos da taxionomia de Bloom é uma descrição de comportamento ou desempenho que se espera do aprendiz. Bloom estava interessado em desenvolver uma taxionomia especifica para avaliar comportamentos dos alunos diante situações de aprendizagem. Para tanto sua taxionomia é formada por três domínios principais: Cognitivo, Afetivo e Psicomotor.

Para o Domínio Cognitivo foram estabelecidos objetivos ligados a memória e ao desenvolvimento de habilidades e capacidades intelectuais. O Domínio Afetivo busca objetivos que descrevem mudança de interesse, atitudes e valores. O Domínio Psicomotor está ligado as habilidades manipulativas e motoras do aprendiz.

Durante anos a taxionomia de Bloom foi utilizada no ambiente educacional para elaboração e avaliação de cursos, propostas pedagógicas, projetos no ensino superior e na educação básica. Com o passar dos anos e com as mudanças ocorridas na sociedade, principalmente na educação, a taxionomia precisou ser revisada, concentrando-se na construção de novos objetivos para o Domínio Cognitivo e na categorização da dimensão conhecimento em:

- O conhecimento efetivo refere-se a um conhecimento básico já conhecido pelo aluno, que possui habilidades e facilidade para lidar com ele.

- O conhecimento conceitual esta apoiado na inter-relação entre elementos isolados de informação, formando um sistema de informação mais elaborado. Este conhecimento pode ser relativo a: teorias, generalização, modelos e classificação.

- O conhecimento procedimental inclui procedimentos, referem-se às técnicas e métodos para serem utilizados em procedimentos de análise e de resolução etc. 
- No conhecimento metacognitivo ocorre uma reflexão sobre o conhecimento adquirido, levando o aluno a pensar em como proceder à manipulação deste processo.

As categorias da dimensão processo cognitivo criando por Bloom foram nomeadas em Lembrar, Entender, Aplicar, Analisar, Avaliar e Criar. Analisaram-se essas categorias a partir dos objetivos para o ensino de matemática propostos pelo PCN.

Lembra consiste em recordar conceitos básicos, isso nos remete a ideia de uma revisão de conteúdo, que objetiva trazer na memória, informações, procedimentos ou conceitos já estudados. Em relação a esta revisão o PCN (1998) afirma:

De modo geral, os professores avaliam que os alunos vêm do ciclo anterior com um domínio de conhecimentos muito aquém do desejável e acreditam que, para resolver o problema, é necessário fazer uma retomada dos conteúdos. [...] É fundamental diagnosticar o domínio que cada aluno tem sobre os diferentes conteúdos que serão explorados. [BRASIL, 1998, p. 61 e 62.]

A revisão, sobre esta perspectiva, se torna um processo onde o aluno será capaz de reconhecer os conteúdos e criar condições de reproduzi-lo posteriormente. O conhecimento dentro da categoria "Lembrar" se define como Factual ou Efetivo, que caracteriza pela relação dos conteúdos básicos que o estudante deve dominar e utilizar.

A categoria entender refere-se às habilidades de interpretação. Nesta etapa há uma conexão entre o que já sabe e o conhecimento novo, utilizando exemplificação, classificações e resumo para realizar comparações e conclusões. Para o PCN (1998),

O estímulo à capacidade de ouvir, discutir, escrever, ler ideias matemáticas, interpretar significados, pensar de forma criativa, desenvolver o pensamento indutivo/dedutivo, é o caminho que vai possibilitar a ampliação da capacidade para abstrair elementos comuns a várias situações, para fazer conjecturas, generalizações e deduções simples como também para o aprimoramento das representações. [BRASIL, 1998, p. 63].

A interpretação possibilita que o aluno utilize suas próprias palavras para apresentar um conteúdo ou conceito. Entender esta relacionada ao conhecimento conceitual, que estabelece uma relação entre elementos básicos em um contexto mais elaborado. Esses elementos serão abordados através de classificação, teorias e modelos.

A aplicação também esta relacionada com o conhecimento conceitual, neste momento espera-se que o aluno seja capaz de distinguir e utilizar de uma estrutura ou procedimento aprendido para aplicar em situações novas. O procedimento utilizado vai depender do conhecimento do aluno e da especificidade da questão ou problemática.

A análise consiste na habilidade de dividir informações em partes e verificar como essas partes se relacionam. Neste sentido o PCN (1998) afirma:

É fundamental desenvolver um trabalho sistematizado de cálculo que inclua a construção e análise de vários procedimentos, tendo em vista que eles relacionam-se e complementam-se. $O$ cálculo escrito, para ser compreendido, apoia-se no cálculo mental, nas estimativas e aproximações. [BRASIL, 1998, p. 114].

Durante a análise diferentes processos serão comparados, seja em partes ou de forma geral, esta ação caracteriza o conhecimento procedimental e metacognitivo, referindo-se ao "como fazer" e à reflexão sobre "o que se sabe fazer". 
$\mathrm{Na}$ categoria avaliação espera-se do aluno a habilidade de criticar, verificar e argumentar sobre aquilo que foi aprendido. Segundo o PCN (1998):

A argumentação está fortemente vinculada à capacidade de justificar uma afirmação e, para tanto, é importante produzir alguma explicação, bem como justificá-la. Assim, um argumento será aceito se for pertinente, ou seja, se ele estiver sustentado por conteúdos matemáticos. [BRASIL, 1998, p. 71].

A avaliação desenvolve no aluno um espírito crítico, capaz de questionar, debater e argumentar. A avaliação, assim como a análise esta relacionada ao conhecimento procedimental e metacognitivo.

Na última categoria, o aluno está propício a criar algo novo, determinar uma solução nova ou diferente. Criar envolve conhecimentos de princípios e generalizações, teoria e estruturas, que estão associados ao conhecimento conceitual. Durante a criação ocorre uma relação entre os elementos mais simples aprendido ate os mais complexos.

Através desta análise das categorias do domínio cognitivo e dos objetivos elaborados pelo PCN de matemática estabeleceram-se os objetivos que serão utilizados para determinar características importantes do material audiovisual. Os objetivos expressam habilidades que estão associadas a uma característica própria do vídeo, essa característica o define a partir de sua finalidade.

\section{O Experimento com Vídeos}

Existe na web um grande acervo de vídeos destinados ao ensino de conteúdos matemáticos a disponibilidade dos professores e alunos. Para a construção da taxionomia de vídeos específicos da matemática, procurou-se levantar características que compõe este material audiovisual. Para tanto, realizou-se um experimento com seis vídeos disponibilizado pelo canal YouTube referentes a conteúdos curriculares de matemática do Ensino Fundamental II. Esses vídeos foram apresentados para seis entrevistados diferentes, um vídeo para cada entrevistado e todos eram professores de Matemática para o Ensino Fundamental II. A Tabela 1 apresenta alguns dos resultados dessa pesquisa.

Tabela 1. Resultados da pesquisa inicial

\begin{tabular}{|l|c|c|c|c|c|c|}
\hline \multicolumn{1}{|c|}{ Características } & vídeo 1 & vídeo 2 & vídeo 3 & vídeo 4 & vídeo 5 & vídeo 6 \\
\hline Narrado & Sim & Sim & Sim & Sim & Não & Sim \\
\hline Personagem & Não & - & Não & Não & - & - \\
\hline Som adequado & Sim & Sim & Não & Sim & Sim & Sim \\
\hline Tempo em minutos & $109: 14$ & $15: 50$ & $09: 52$ & $08: 40$ & $06: 36$ & $15: 59$ \\
\hline Imagens /desenho & Não & Sim & Não & Sim & Não & Não \\
\hline Legenda & Não & Sim & Não & Não & Não & Não \\
\hline Destaque de palavras & Sim & Sim & Não & Não & Não & Não \\
\hline Apresenta o objetivo da aula/vídeo & Não & Sim & Não & Não & Não & Não \\
\hline Apresenta conteúdo & Não & Sim & Não & Sim & Não & Não \\
\hline Revisa conteúdo anterior & Sim & Sim & Não & Não & Não & Sim \\
\hline Conteúdo a partir de uma situação & Não & Sim & Não & Sim & Não & Não \\
\hline Apresenta definição & Sim & Não & Sim & Sim & Não & Sim \\
\hline Exemplos & Sim & 1 para cada & Sim & Sim & Sim & Sim \\
\hline Exemplos com níveis diferentes & Sim & Não & Sim & Não & Não & Não \\
\hline Exemplos contextualizados & Sim & Sim & Não & Sim & Não & Não \\
\hline Problemática & Não & Sim & Não & Sim & Não & Não \\
\hline Apresenta regras ou métodos & Sim & Sim & Sim & Sim & Não & Não \\
\hline Apresenta exercício & Sim & Não & Sim & Não & Não & Não \\
\hline Apresenta passo a passo a resolução & Sim & Sim & Sim & Não & Sim & Sim \\
\hline
\end{tabular}


Ao assistir os vídeos e analisá-los evidenciaram características referentes à exposição e abordagem do conteúdo e elementos estéticos de apresentação. Dividiramse as características encontradas em três categorias: Aspectos pedagógicos do conteúdo, Aspectos pedagógicos da mídia e Aspectos técnicos da mídia.

$\mathrm{Na}$ categoria aspectos pedagógicos do conteúdo evidenciaram características dos elementos de apresentação do conteúdo específico da matemática, onde foi observado se a apresentação surge a partir da definição/conceituação ou de uma situação.

Outra observação foi à exposição de exemplos, analisando os variados níveis, a contextualização e a problemática envolvida. Segundo o PCN, "o ensino-aprendizagem de Matemática tem como ponto de partida a resolução de problemas" [Brasil, 1998, p. 56]. Desta Forma, verificou-se como a problemática é apresentada, seja por meio de exercícios resolvidos ou que irão resolver ao longo do vídeo. Preocupou- se em observar também, se o vídeo propõe ou sugere exercícios para que o aluno resolva.

$\mathrm{Na}$ categoria aspectos pedagógicos da mídia, procurou-se por característica dos vídeos que seja utilizada em caráter pedagógico e que venha influenciar na apresentação do conteúdo. Analisou-se no experimento o cenário onde o vídeo foi feito, sua narração, apresentação de legendas, destaques de palavras, etc. A categoria aspectos técnicos da mídia envolve características próprias do vídeo. Verificou a adequação do som, imagem, gravação, tempo de duração, dentre outros.

Com este experimento visualizou-se inicialmente características relevante nos vídeos que são fundamentais ou não para o ensino e a aprendizagem da matemática.

\section{Proposta de Taxionomia de Vídeos}

As características consideradas no experimento citado na seção anterior, permitiu dividir as categorias do vídeo em três aspectos: Técnico da Mídia, Pedagógico da Mídia e Pedagógico do Conteúdo. Ao analisar os aspectos pedagógicos da mídia, percebeu-se a existência de características pessoais e subjetivas, sem influência na análise, o que impossibilitaria uma avaliação generalizada pelos professores. Devido a isso retiramos os aspectos pedagógicos da mídia da taxionomia.

Os aspectos Técnicos da Mídia e os Pedagógicos do conteúdo serão apresentados como categorias da taxionomia constituídos de características próprias. As estruturas dos vídeos giram em torno desses aspectos principais e para utilização no contexto educacional damos ênfase na categoria pedagógico do conteúdo.

$\mathrm{Na}$ categoria aspectos Técnicos da Mídia evidenciam características específica e técnicas do material audiovisual.

- Qualidade de Imagem: Na qualidade da imagem está presente toda estrutura do vídeo, sua estética, composição e utilização. Nesta condição, o professor precisar analisar se os elementos visuais que compõem o vídeo estão visíveis, se sua apresentação atende aquilo que foi proposto demonstrar.

- Qualidade de áudio: O som é complementar a imagem no vídeo, ele em muito dos casos representa aquilo que a imagem esta caracterizando. Um som adequado possibilita a quem assiste ao vídeo ter uma compreensão daquilo que esta transmitindo. 
- Narração: O áudio é uma das formas de comunicação com os alunos. Sua apresentação poderá ser narrada, sendo o locutor oculto, narrado com personagens executando as ações, ou narrado pelos personagens.

- Organização: Corresponde à apresentação da interação simultânea de som e imagem, imagem e texto. Nesta característica o professor deverá analisar se estes elementos estão se relacionando de acordo com a apresentação.

- Forma de disponibilização: O formato em que o vídeo esta disponível é uma característica importante, pois condiz com a forma com que o aluno vai assistir ao vídeo. Esse formato pode ser em CD, DVD, através de sites na internet, etc. Envolve também o tipo de extensão e codificação do vídeo, exemplo: mp4 com codec h-264.

- Acessibilidade: É uma característica que poucos vídeos apresentam. Constitui da disposição de legendas para um público específico com algumas necessidades especiais.

- Tempo de duração do vídeo: Este deve ser analisado pelo professor para diagnosticar se o objetivo do vídeo foi alcançado dentro do tempo de produção, bem como se o tempo em questão atende as especificidades dos alunos a quem se destina.

Algumas características técnicas do vídeo não foram apresentadas na taxionomia por considerar que seria necessário um conhecimento maior e específico do professor para serem avaliadas.

A categoria aspectos Pedagógicos do Conteúdo evidenciam características fundamentais para a apresentação de um conteúdo da matemática. Essas características estão relacionadas com a finalidade do vídeo, ou seja, elas vão ser apresentadas a partir daquilo que se objetiva com o material. São elas:

- Apresentar objetivo: constitui-se de uma abordagem resumida do conteúdo do vídeo, ou seja, aquilo que o vídeo pretende apresentar como um todo.

- Revisão do conteúdo anterior: esta caracterizada em um retorno de ideias principais que serão fundamentais para apresentar o novo conteúdo.

- Apresentação de um conteúdo específico da matemática: caracteriza-se em explanação de ideias, princípios e teorias que compõem o conteúdo. A definição também envolve teorias e conceitos, são informações que irão formalizar matematicamente o conteúdo que se objetiva apresentar.

- Exemplos: são características importantes dentro dos aspectos pedagógicos do conteúdo. Eles se apresentam como a explanação de aplicação do conteúdo, podendo ser abordados a partir de diferentes níveis e perspectivas evidenciando a contextualização ou a interdisciplinaridade. Têm o objetivo de enriquecer o conteúdo e possibilitar a visualização de aplicação deste conteúdo em uma problemática.

- Regras e métodos: representa a inserção ou destaque de procedimento de cálculos, metodologias de resoluções e técnicas que favorecem soluções. Esta apresentação poderá vim através do exemplo ou da problemática. 
- Representações: constituem-se de tabelas, gráficos, diagramas, importantes para organização de dados, auxiliando na interpretação, análise e conclusão. A presença destas representações vai depender do conteúdo matemático abordado.

- Exercícios: têm o caráter de retornar aquilo que foi apresentado como conteúdo. Nos vídeos os exercícios podem aparecer já resolvidos na tela explicando somente o que foi feito ou podem ser resolvidos passo a passo.

Com essas características definiram-se alguns tipos de vídeos baseado na sua finalidade. Dividiram-se os vídeos voltados para o ensino de matemática em: Introdução e Apresentação de conteúdo, Aplicação de conteúdo específico em situações variadas (exemplos e/ou exercícios), Fixação ou Reforço de conteúdo.

Nos vídeos destinados a introdução e apresentação de conteúdo evidenciaram características que apresenta este objetivo. Nesses vídeos predominam a exposição sistemática de conteúdo, abordada geralmente através de exemplos. Apresenta-se também a definição do conteúdo através de linguagens matemática.

O processo cognitivo para vídeos com a finalidade de introduzir e apresentar conteúdo é o "Lembrar" e "Entender". Cada ação constituída por estes processos cognitivos, embasado pelo PCN (1998), esta associada a uma característica do vídeo.

A Tabela 2 apresenta as características dos vídeos que introduzem e apresentam conteúdos e foi elaborada com base no processo do domínio cognitivo lembrar e entender da taxionomia de Bloom.

Tabela 2. Características de vídeos com finalidade de introduzir e apresentar conteúdos.

\begin{tabular}{|c|c|c|c|}
\hline Tipo & \multicolumn{2}{|c|}{ Aspectos Pedagógicos do Conteúdo } & Objetivos PCN \\
\hline \multirow{7}{*}{ 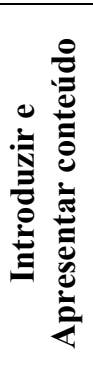 } & \multicolumn{2}{|c|}{ Objetivo da aula/vídeo } & \\
\hline & \multicolumn{2}{|c|}{ Revisão do conteúdo ant } & Reconhecer representações e traduzir situações problemas \\
\hline & \multicolumn{2}{|c|}{ Apresentação do conteúdo } & Ampliar, consolidar e construir novos significados. \\
\hline & \multicolumn{2}{|c|}{ Conteúdo a partir de uma situação } & $\begin{array}{l}\text { Resolver situações problemas reconhecendo e ampliando } \\
\text { os conceitos e definições que o cercam }\end{array}$ \\
\hline & \multicolumn{2}{|l|}{ Definição } & Traduzir informações generalizando regularidades \\
\hline & \multirow[b]{2}{*}{ Exemplos } & Níveis diferentes & Identificar, interpretar e utilizar diferentes representações. \\
\hline & & Contextualizados & $\begin{array}{l}\text { Reconhecer informações e organiza-las em representações } \\
\text { matemáticas }\end{array}$ \\
\hline
\end{tabular}

Ao selecionar ou avaliar vídeos desta natureza, o professor buscará neste material um conteúdo que levará o aluno a recordar, ampliar, consolidar e construir novos conhecimentos a partir do conteúdo matemático apresentado.

Os vídeos destinados à aplicação de conteúdo específico em situações variadas (exemplos e/ou exercícios) apresentam características de exposição de um conteúdo matemático aplicado em exemplos ou exercícios. Essas características, vide Tabela 3, foram associadas ao processo cognitivo da taxionomia de Bloom: "Entender", "Aplicar" e "Analisar". Este tipo de vídeo constitui de objetivos voltados para aplicação de um conhecimento novo adquirido pelo aluno, evidenciando habilidades de utilizar diferentes procedimentos e técnicas de resolução, dividir o conteúdo e relacionar suas partes, envolvendo observação, análise, interpretação, dentre outras ações. 
Tabela 3: Características de vídeos com a finalidade de Aplicação de conteúdo

\begin{tabular}{|c|c|c|c|}
\hline Tipo & \multicolumn{2}{|c|}{ Aspectos Pedagógicos do Conteúdo } & Objetivos PCN \\
\hline \multirow{9}{*}{ 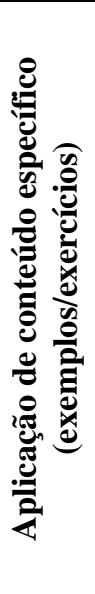 } & \multicolumn{2}{|c|}{ Apresentação de conteúdo } & Utilizar e construir novo conhecimento \\
\hline & \multicolumn{2}{|c|}{ Conteúdo a partir de uma situação } & $\begin{array}{l}\text { Resolver situações problemas e a partir dela ampliar e } \\
\text { construir novos significados }\end{array}$ \\
\hline & \multicolumn{2}{|c|}{ Apresentação de Problemática } & Resolver situações problemas utilizando estratégias \\
\hline & \multirow[b]{2}{*}{ Exemplo } & Níveis diferentes & Produzir e interpretar diferentes linguagens \\
\hline & & Contextualizado & $\begin{array}{l}\text { Estabelecer relações e construir interpretações das } \\
\text { representações matemáticas }\end{array}$ \\
\hline & \multicolumn{2}{|c|}{$\begin{array}{l}\text { Representações: Tabelas, gráficos, } \\
\text { diagramas. }\end{array}$} & $\begin{array}{l}\text { Construir representações, elaborar conclusões, analisar e } \\
\text { interpretar essas representações. }\end{array}$ \\
\hline & \multicolumn{2}{|c|}{ Apresenta regras e métodos } & Selecionar e utilizar diferentes procedimentos de cálculo \\
\hline & \multirow{2}{*}{ Exercício } & $\begin{array}{l}\text { Resolução passo a } \\
\text { passo }\end{array}$ & $\begin{array}{l}\text { Resolver situações problemas compreendendo os } \\
\text { procedimentos envolvidos }\end{array}$ \\
\hline & & Resolução na tela & $\begin{array}{l}\text { Analisar situações problemas identificando elementos e } \\
\text { desenvolvendo conceitos. }\end{array}$ \\
\hline
\end{tabular}

Vídeos com esta finalidade diferem do anterior pela aplicação do conteúdo, na exploração, seleção e utilização de métodos e procedimentos de resoluções, também se exploram as representações algébricas, geométricas e as de organização de dados.

Os vídeos para fixação ou reforço de conteúdo exploram exemplos e exercícios. Espera-se do aluno habilidades de resolução, aprimorando técnicas através de exemplos e exercícios diversificados. Os domínios cognitivos da taxionomia de Boom relacionados a este tipo de vídeo são: "Entender", "Aplicar", "Analisar" e "Avaliar". A relação está apresentada pela Tabela 4. Os exemplos e exercícios exigirão observação e leitura do contexto, das relações matemáticas envolvidas e das estratégias de resolução, também exigirá do aluno a análise de informações, a construção de argumentação e conclusão.

Tabela 4: Características de vídeos com a finalidade de Fixação ou Reforço

\begin{tabular}{|c|c|c|c|}
\hline Tipo & \multicolumn{2}{|c|}{ Aspectos Pedagógicos do Conteúdo } & Objetivos PCN \\
\hline \multirow{6}{*}{ 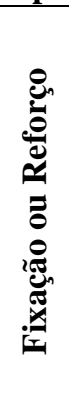 } & \multirow[b]{2}{*}{ Exemplo } & Níveis diferentes & Produzir e interpretar diferentes linguagens \\
\hline & & Contextualizados & $\begin{array}{l}\text { Estabelecer relações e construir interpretações das } \\
\text { representações matemáticas }\end{array}$ \\
\hline & \multicolumn{2}{|c|}{ Possuem tabelas, gráficos, diagramas. } & $\begin{array}{l}\text { Construir representações, elaborar conclusões, analisar, } \\
\text { interprestar essas representações. }\end{array}$ \\
\hline & \multicolumn{2}{|c|}{ Apresenta regra e método } & Selecionar e utilizar diferentes procedimentos de cálculo \\
\hline & \multirow{2}{*}{ Exercício } & Resolução passo a passo & $\begin{array}{l}\text { Resolver situações problemas compreendendo os } \\
\text { procedimentos envolvidos }\end{array}$ \\
\hline & & Resolução na tela & $\begin{array}{l}\text { Analisar situações problemas identificando elementos e } \\
\text { desenvolvendo conceitos }\end{array}$ \\
\hline
\end{tabular}

As Tabelas 2, 3 e 4 distinguiram-se através da categoria aspectos pedagógicos do conteúdo pela diferença entre os tipos de vídeos. Suas características vão ao encontro de suas finalidades ao se relacionar com ações do processo cognitivo.

A Figura 1 apresenta um organograma da Taxionomia proposta de vídeos para a Matemática. $\mathrm{O}$ organograma é o resumo organizado das tabelas apresentadas com as categorias e suas respectivas características referentes à taxionomia proposta. 


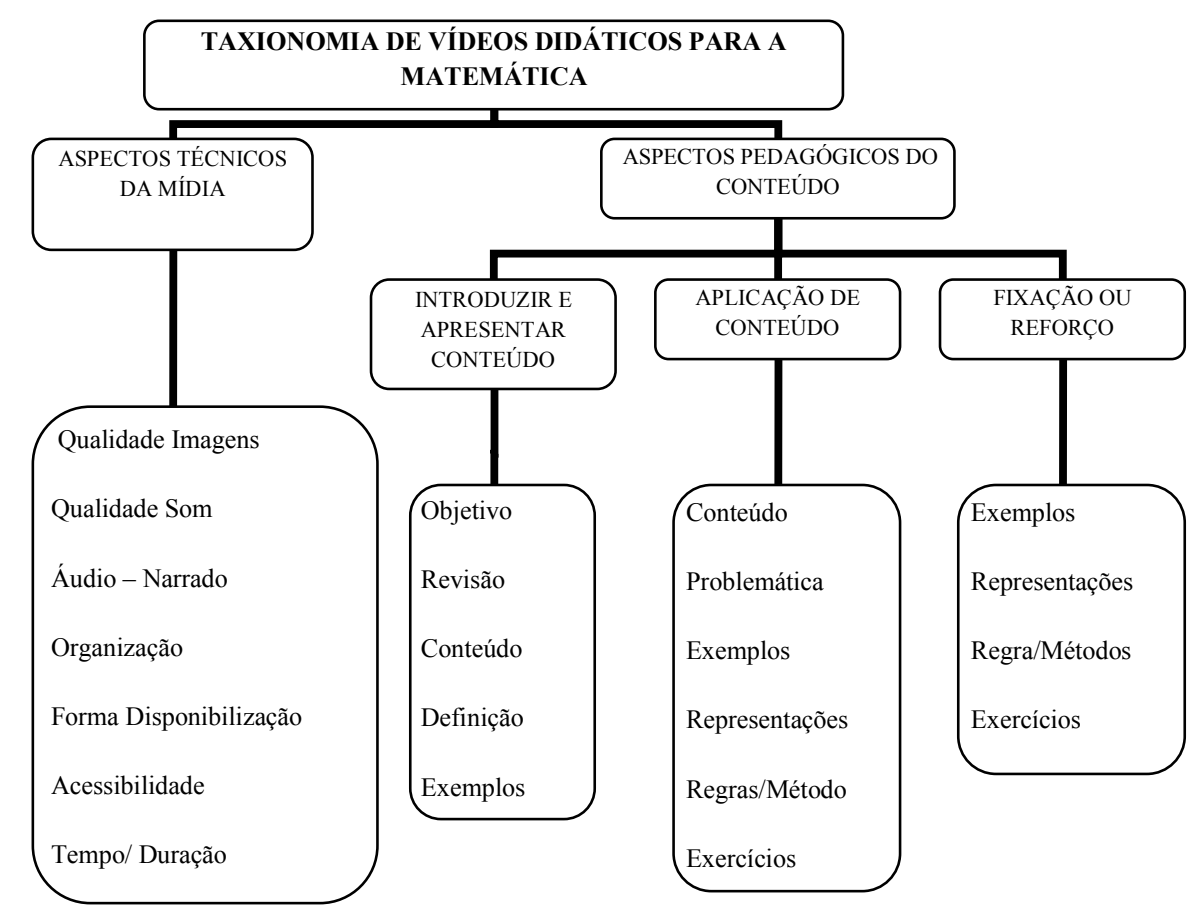

Figura 1. Organograma da Taxonomia de Vídeos para Matemática

\section{Considerações Finais}

O vídeo no ensino de matemática além de mudar o cenário das aulas, pode desenvolver no aluno a percepção em relação aos conteúdos e conceitos, abordados através das diversas linguagens, despertando a construção de ideias e argumentos.

A seleção e avaliação do vídeo são de grande importância para sua utilização com os alunos, entretanto, o professor necessita de informações a respeito deste material para proceder ao julgamento. Desta forma apresentou-se a proposta de taxionomia de vídeos para apoiar o professor de matemática na seleção e avaliação de vídeos destinados a alunos do Ensino Fundamental II.

Para construção da taxionomia proposta recorreu-se aos objetivos descritos pelo PCN de Matemática e a taxionomia de Bloom. Foi explorada a dimensão conhecimento, que evidenciou um processo com diferentes níveis de abordagens e as categorias da dimensão processo cognitivo. Na taxionomia proposta as características foram apresentadas desde ação de aprendizagem que constitui em "lembrar" até "criar".

Espera-se na seleção do vídeo, que o professor analise as características presente nas tabelas 1,2 e 3 e as habilidades que o material irá proporcionar baseado em ações que descrevem os objetivos a serem adquirido pelos alunos através do vídeo.

Nem todas as características definidas na categoria aspectos pedagógicos do conteúdo poderão ser encontradas em um único material, entretanto as características apresentadas são a base para seleção, pois determinam a finalidade do vídeo. Optou-se somente por essas características por entender que elas já constituem de características importantes e uma taxionomia extensa inviabiliza a avaliação e seleção do professor. 


\section{Referências}

Brasil, Secretaria de Educação. (1998) "Parâmetros Curriculares Nacionais: Ensino Fundamental". Brasília: MEC.

Ferrés, j. (1996) "Vídeos e educação". Porto Alegre: Editora Artes Médicas.

Gomes, L. F. (2008) "Vídeos Didáticos: uma proposta de critérios para analise". Revista brasileira Est. Pedag., Brasília, v.89, n. 223, p.477-492. Disponível em <http://rbep.inep .gov.br/index.php/RBEP/article/view/684/1153>. Acesso em 04 fev. 2014.

Moram, J. M. (2005) "Integração das tecnologias na educação. Desafios da televisão e do vídeo à escola”. Secretaria de Educação a Distância, SEED. Disponível em $<$ http:// tvescola.mec.gov.br/images/stories/... para.../livro_salto_tecnologias.pdf $>$ Acesso em 10 Abr 2014.

Prata, C. L.; Nacimento, A. C. A. A. (2007) "Objetos de Aprendizagem: uma proposta de recurso pedagógico". MEC. Secretaria de Educação à distância. Brasil.

Silva, A. M. (2011) "O vídeo como recurso didático no ensino da matemática”. 198 f. Dissertação (Mestrado em Educação em Ciências e Matemática) Universidade Federal de Goiás. PrPPG, Goiás. 